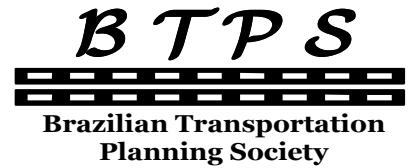

Planning Society

\author{
Journal of Transport Literature \\ Vol. 7, n. 4, pp. 175-198, Oct. 2013 \\ Research Directory
}

\section{JTL|RELIT}

www.transport-literature.org ISSN 2238-1031

\title{
Análise das principais métricas utilizadas no zoneamento acústico de áreas próximas a aeródromos
}

[Analysis of key metrics used in sound zoning around of areas close to aerodromes]

\author{
Edson Benício de Carvalho Júnior*, Sérgio Luiz Garavelli, Felipe Vivian Smozinski, Armando \\ de Mendonça Maroja, Wesley Candido de Melo
}

\begin{abstract}
Universidade Católica de Brasília - Brazil, Universidade Católica de Brasília - Brazil, Universidade Católica de Brasília - Brazil,
\end{abstract} Universidade de Brasília - Brazil, Universidade Católica de Brasília - Brazil

Submitted 10 Sep 2012; received in revised form 20 Jan 2013; accepted 22 Jan 2013

\begin{abstract}
Resumo
Esse trabalho possui por objetivo apresentar uma análise comparativa entre as principais normas e legislações que avaliam o ruído aeronáutico. A comparação buscou avaliar as metodologias e as métricas acústicas utilizadas no contexto brasileiro. Experiências internacionais também foram consideradas. Da análise realizada, verifica-se a existência de divergências entre as legislações, principalmente com relação às métricas empregadas para a monitoração e caracterização do nível de incômodo sonoro gerado pela operação de aeronaves. No Brasil, consolida-se o uso da métrica que indica o nível de pressão sonora dia noite (Ldn). Destaca-se que os países estudados utilizam mais de uma métrica para avaliar o ambiente sonoro de regiões vizinhas a aeródromos. A realização de estudos com outras métricas, no contexto brasileiro, pode auxiliar no planejamento do uso e ocupação do solo no entorno dos aeroportos. Por fim, entende-se que essa pesquisa contribui para um melhor entendimento das métricas atualmente empregadas para a avaliação do ruído aeronáutico. Além disso, também pode contribuir para o aprimoramento dos planos de zoneamento de ruído em aeródromos.
\end{abstract}

Palavras-Chave: ruído aeronáutico, índice ponderado de ruído, nível de incômodo sonoro, normas técnicas, comunidades.

\begin{abstract}
The aim of this paper is to present a comparative analysis between the standards that evaluate aircraft noise. The comparison evaluates the methodologies and acoustics metrics used in Brazilian context. International experiences have also been considered. The analysis indicates the existence of differences between the standards, particularly with respect to the metrics used to monitor and characterize the level of noise annoyance causing by aircraft operations. Brazil has consolidated the use of metric that indicates the sound pressure level day - night (Ldn). Other countries use more than one metric to assess the soundscape in the vicinity at the airports. The studies with other metrics for the Brazilian context can assist in planning the use and occupation of land around airports. Finally, this research contributes to a better understanding of the metrics currently used for the assessment of aircraft noise. Furthermore, can also contribute to the improvement the sounding zoning around of areas close at the aerodromes.
\end{abstract}

Key words: aircraft noise, weighted noise index, annoying sound level, technical standards, communities.

*Email: edsonbenicio@gmail.com.

\section{Recommended Citation}

Carvalho Jr, E. B., Garavelli, S. L., Smozinski, F. V., Maroja, A. M. and Melo, W. C. (2013) Análise das principais métricas utilizadas no zoneamento acústico de áreas próximas a aeródromos. Journal of Transport Literature, vol. 7, n. 4, pp. 175-198.

- JTL/RELIT is a fully electronic, peer-reviewed, open access, international journal focused on emerging transport markets and published by BPTS - Brazilian Transport Planning Society. Website www.transport-literature.org. ISSN 2238-1031. 


\section{Introdução}

Esse estudo apresenta uma análise comparativa entre as principais normas e legislações que avaliam o ruído proveniente da operação de aeronaves. A análise teve como referência o contexto brasileiro, mas experiências internacionais também foram consideradas com o intuito de determinar semelhanças, diferenças ou relação entre as estratégias adotadas entre os países para o monitoramento e controle do ruído aeronáutico.

Essa modalidade de ruído é uma das principais externalidades negativas relatadas em áreas residenciais próximas a aeródromos. Isso se deve ao fato da operação de uma aeronave gerar barulho facilmente identificável e que frequentemente impacta o meio ambiente com energia sonora excessiva (Group, 2003). No Brasil e no mundo, estudos têm indicado um crescimento considerável no número de reclamações por parte da população em relação ao ruído gerado nos grandes centros urbanos (Carvalho Jr., E.B, Garavelli, S.L. e Maroja, A.M., 2012).

O incômodo sonoro causado às comunidades próximas a aeroportos configura-se em um dos principais impactos causados pelas operações aeroviárias. Além de provocar irritação contribui diretamente para o desenvolvimento de doenças como hipertensão, problemas cardíacos, males associados a distúrbios no sono, psicológicos, emocionais e estresse. O HYENA (HYpertension and Exposure to Noise near Airports), foi um grande estudo realizado simultaneamente em seis países europeus onde investigou-se a associação entre ruído aeroportuário, tráfego urbano e pressão sanguínea incluindo hipertensão. Os resultados indicaram um grande risco de desequilíbrio cardiovascular em sujeitos expostos a altos níveis de ruído, sendo que pessoas expostas a um nível de pressão sonora maior ou igual a 70 decibels por longos períodos estão sujeitos a efeitos crônicos de estresse causados pelo ruído (Jarup et al., 2005).

O HYENA (HYpertension and Exposure to Noise near Airports), foi um grande estudo realizado simultaneamente em seis países europeus onde investigou-se a associação entre ruído aeroportuário, tráfego urbano e pressão sanguínea incluindo hipertensão. Os resultados indicaram um grande risco de desequilíbrio cardiovascular em sujeitos expostos a altos níveis de ruído, sendo que pessoas expostas a um nível de pressão sonora maior ou igual a 70 
decibels por longos períodos estão sujeitos a efeitos crônicos de estresse causados pelo ruído (Jarup et al., 2005).

No que diz respeito especificamente ao ruído de aeronaves, um dos resultados mais expressivos do HYENA indica que o incômodo das pessoas com o ruído aeroviário aumentou ao longo dos últimos anos. O estudo ainda sugere que as atuais metodologias relacionadas à determinação da porcentagem de pessoas incomodadas, ou altamente incomodadas, com o ruído dos aviões, sejam revisadas (Babisch, W. et al., 2009).

O incômodo sonoro induzido pelo ruído aeroviário destaca-se não só por provocar reações adversas nas pessoas afetadas, mas também por possuir potencial de restringir a operação de um aeroporto, com implicações na sustentabilidade financeira do aeródromo. É o caso do Aeroporto Internacional de Congonhas, em São Paulo, onde as comunidades vizinhas manifestaram-se contra o aumento do tráfego aéreo levando ao fechamento noturno do aeroporto.

Sendo assim, fica destacada a importância de se ter normas e legislações capazes de garantir uma metodologia confiável e precisa a respeito da determinação dos impactos do ruído aeroviário. O intuito é o de evitar, prevenir ou reduzir prioritariamente os efeitos prejudiciais da exposição ao ruído.

Dessa forma, no presente trabalho realizou-se uma análise comparativa entre as diversas legislações, nacionais e internacionais, que versam sobre o ruído aeronáutico. Também se efetuou uma pesquisa a respeito das principais métricas acústicas utilizadas no mundo para avaliação do ruído aeronáutico.

Desenvolveu-se pesquisa bibliográfica que permitiu investigar as métricas acústicas empregadas bem como as metodologias e recomendações previstas nas normas e portarias brasileiras, a destacar: NBR 10.151, NBR 10.856, NBR 11.415, NBR 12.859, NBR 13.368, portaria $\mathrm{n}^{\circ}$ 1.141/GM5/87 e resolução CONAMA n 01/1990 que considera a poluição sonora uma fonte de deterioração da qualidade de vida.

Em seguida, realizou-se uma revisão a respeito das normas e métricas utilizadas em diversos países, entre eles Estados Unidos, Canadá, Índia, África do Sul e Comunidade Européia. 
Como método de análise buscou-se comparar, nas diversas normas e legislações, as metodologias e métricas acústicas empregadas.

Essa comparação permitiu identificar a existência de abordagens comuns, ou diferentes, adotadas pelos países estudados com relação à avaliação do ruído aeronáutico. Pode-se antecipar que verificou-se divergências entre as normas e portarias analisadas, principalmente com relação à métrica acústica a ser utilizada para monitoração e caracterização do nível de incômodo sonoro gerado por tráfego aéreo.

Assim, esse estudo contribui para um melhor entendimento das principais métricas acústicas adotadas, atualmente, para a avaliação do impacto sonoro causado pelo ruído aeroviário em áreas circunvizinhas a aeroportos. Além disso, nessa pesquisa foi possível identificar as possíveis contradições e relações entre as diversas legislações, o que pode contribuir para o aprimoramento dos planos de zoneamento de ruído em aeródromos. Dessa forma, pode-se proporcionar a inserção de atividades aeroportuárias menos conflituosas e que assegurem condições adequadas de relacionamento entre os operadores de um aeroporto e a comunidade.

Por fim, o trabalho é composto das seguintes seções: Seção 1 encontra-se a descrição das principais métricas acústicas utilizadas para avaliação do ruído aeronáutico. Já a Seção 2 é composta pela análise das normas, portarias e legislações, nacionais e internacionais. A Seção 3 apresenta a análise dos resultados e por fim, têm-se as conclusões.

\section{Principais métricas acústicas utilizadas para avaliação do ruído aeronáutico}

\section{$1.1 L_{e q}$ (Nível de pressão sonora equivalente)}

O nível contínuo equivalente é o som produzido durante um dado período de tempo medido com equipamento adequado e calibrado com filtro de frequências na ponderação "A". Nível de pressão sonora equivalente, $\mathrm{L}_{\mathrm{eq}}$, em dB(A), é calculado pela Equação (Gerges, 2000):

$$
L_{e q}=10 \times \log _{10}\left(\frac{1}{T} \int_{0}^{T} \frac{p(t)^{2}}{p_{0}^{2}} d t\right)
$$


Onde T é a duração do período de referência (tempo total de medida); $\mathrm{P}(\mathrm{t})$ é a pressão sonora instantânea; $\mathrm{P}_{0}$ é pressão sonora de referência $\left(2,0 \times 10^{-5} \mathrm{~N} / \mathrm{m}^{2}\right)$. A Equação 1 mostra que o nível equivalente é representado por um valor constante que durante o mesmo tempo T, resultaria na mesma energia acústica produzida pelos valores instantâneos variáveis de pressão sonora.

\subsection{Nível de Ruído Percebido (PNL) e Nível de Ruído Percebido com Correção de Tom (PNLT)}

Segundo a NBR 11.415 (ABNT, 1990), o PNL é o resultado do cálculo dos contornos de igual índice de ruidosidade, de frequências de banda larga e tipos de ruído produzidos por aeronaves, a pistão ou a jato, no pouso e decolagem convencionais. No cálculo, consideram-se um campo sonoro difuso e a inexistência de irregularidades pronunciadas no espectro. É expresso pela Equação 2.

$$
P N L=\left(10 \times \log _{10} N / \log _{10} 2\right)+40 d B
$$

Onde $\mathrm{N}$ expressa a ruidosidade total em noys. O noy é uma grandeza psicoacústica desenvolvida para mensurar objetivamente o incômodo do ruído e expressa a ruidosidade percebida. A ruidosidade percebida de um tom puro (som em uma única frequência) em 1 kHz, com nível de pressão sonora de $40 \mathrm{~dB}$, equivale a 1 noy. Ruídos de 2, 4, 10 ou 20 noys são considerados com tendo ruidosidade percebida $2,4,10$ ou 20 vezes maior que 1 noy. Portanto, o noy expressa a sensação subjetiva de incômodo do ruído em uma escala linear (BISTAFA, 2006). Sendo assim, o noy é a unidade percebida de um ruído cujo PNL é de 40 $P N d B$ (ABNT, 1990). Ressalta-se que campo sonoro difuso e o campo acústico no qual a energia por unidade de volume tem o mesmo valor em todos os pontos e onde a intensidade acústica é a mesma em todas as direções (ABNT, 1990).

Caso o espectro apresente tons puros expressivos, um refinamento adicional do método consiste na correção para cada valor do PNL obtido. Isso requer que se identifiquem picos relativamente elevados nos espectros medidos, aplicando-se correções conforme regras estabelecidas na norma ISO 3891. Nos piores casos, essa correção poderá ser de até $+7 \mathrm{~dB}$. O resultado é conhecido como nível de ruído percebido com correção de tom (PNLT) (BISTAFA, 2006). Dessa forma, o PNLT é a correção do nível de ruído percebido, acrescido 
de um fator de correção de tom, quando existe ocorrência de irregularidades espectrais (ABNT, 1990). É definido da seguinte forma (ABNT, 1990):

$$
P N L T=(P N L+C)
$$

Onde C indica a correção do tom.

\subsection{Nível Efetivo de Ruído Percebido (EPNL - Effective Perceived Noise Level)}

O EPNL é calculado integrando-se o registro de PNLT no intervalo de tempo durante o qual este esteve até $10 \mathrm{~dB}$ abaixo do valor máximo, sendo resultado normalizado pelo intervalo de tempo de referência (T) de 10s. A ideia por trás da normalização com o valor de 10 s é penalizar aqueles aviões que fazem muito ruído durante muito tempo, considerando-se também que 10s é um tempo razoável para um sobrevoo típico (Bistafa, 2006).

Basicamente o EPNL avalia três propriedades: nível de pressão sonora, distribuição de frequência e variação do tempo (ABNT, 1990). O EPNL é representado pela Equação 4 (NBR 11.415/ABNT 1990 e Bistafa, 2006).

$$
E P N L=10 \times \log _{10}\left[\frac{1}{T} \int_{0}^{t} 10^{[(P N L T(t) / 10]} d t\right]
$$

Onde, PNLT $(t)$ é o nível de ruído percebido com correção de tom em um instante $t$ e T é o tempo de referência equivalente a 10 s.

\subsection{Nível de ruído previsto (NEF - Noise Exposure Forecast)}

O NEF tem por base o EPNL e tem sido usado pelo Departamento de Habitação e Desenvolvimento Urbano dos Estados Unidos (US HUD) e pelo Departamento de Transportes do Canadá (Transport Canada) para zoneamento do ruído no entorno de aeroportos. Esse zoneamento é elaborado em termos de curvas isofônicas, sendo o NEF a grandeza que caracteriza as curvas. É somado a todos os tipos de aeronaves e todos os trajetos de vôo e é definido pela Equação 5 (Levesque, 1994 e Bradley, J.S, 1996). 


$$
N E F=\langle E P N L\rangle+10 \times \log \left(N_{d}+16,7 \cdot N_{n}\right)-88
$$

Onde $\langle E P N L\rangle$ é a média do EPNL de sobrevoo das aeronaves, $N_{d}$ e $N_{n}$ representam o número do tempo correspondente ao período diurno ( $7 \mathrm{~h}$ as $22 \mathrm{~h}$ ) e ao noturno ( $22 \mathrm{~h}$ as $7 \mathrm{~h}$ ), respectivamente. Destaca-se que as curvas isofônicas representam a união de pontos com a mesma avaliação numérica, ou nível de ruído, no entorno de um aeroporto. Leva-se em consideração a possibilidade de se obter os níveis de incômodo, para um determinado período, um dia, por exemplo, obtendo-se um conjunto de contornos, ou curvas, de igual nível de incômodo (Nunes e Sattler, 2004). Por meio dessas curvas, pode-se avaliar a extensão do impacto sonoro produzido pelo aeroporto, além de analisar quantitativamente os efeitos de soluções imaginadas. Dessa forma, pode-se elaborar uma política de ocupação do solo, que harmonize a convivência entre o aeroporto e a comunidade servida (IAC, 1981).

\section{5 Índice Ponderado de Ruído (IPR)}

No Decreto $\mathrm{n}^{\circ}$. 89.431, de 8 de março de 1984, nível de incômodo sonoro é definido como a medida em IPR (Índice Ponderado de Ruído), cumulativa, em escala logarítmica, do incômodo causado pelo ruído gerado pela operação de aeronaves em um aeroporto. Esse decreto dispõe sobre o Plano Básico de Zoneamento de Ruído (PBZR) e Planos Específicos de Zoneamento de Ruído (PEZR) a que se refere o Código Brasileiro do Ar.

Poucos anos depois, o IPR é adotado na portaria n ${ }^{\circ}$. 1.141/GM5 de 1987, e até 28 de setembro de 2011(ver item 3.6) era o método oficial adotado pelo Brasil para o cálculo do incômodo produzido por aeronaves, tendo como referência os contornos IPR 75 e $65 \mathrm{~dB}(\mathrm{~A})$ para as curvas isofônicas (curvas de igual nível de incômodo sonoro) utilizadas no PBZR.

As NBR's 11.415 (ABNT, 1990) e NBR 12.859 (ABNT, 1993) definem o IPR por meio da Equação 6:

$$
I P R_{(i, j, k)}=E P N L_{(i, j, k)}+10 \log \cdot p(k) \cdot n_{(i, j, k)}-68
$$

Onde, $n_{(i, j, k)}$ é o número dessas aeronaves, $p(k)$ é o fator de ponderação em função do período do dia: $p(1)=1$ para o período diurno e $p(2)=10$ para o período noturno (ABNT, 
1990 e ABNT, 1993). Aqui se chama a atenção para os pesos diferenciados entre voos diurnos e noturnos.

Após 1994 a fórmula matemática foi aprimorada com o objetivo de permitir medições diretas em campo, e encontra-se descrito no Airport Planning Manual. É definido como sendo o nível médio de ruído, ponderado na escala "A", para um período de $24 \mathrm{~h}$, aplicando-se um acréscimo de $10 \mathrm{~dB}(\mathrm{~A})$ nos níveis de ruído que ocorrem no período noturno (ICAO, 2002 apud VALIM, 2006). Obter o valor desse índice contribui com o entendimento das possíveis reações das comunidades afetadas pelo ruído aeroviário. A fórmula aprimorada é determinada pela expressão 7 .

$$
I P R=10 \times \log \left[\frac{1}{24}\left(15 \times 10^{\frac{L_{d}}{10}}+9 \times 10^{\frac{\left(L_{n}+10\right)}{10}}\right)\right]
$$

Nesta expressão, o número 24 corresponde às horas medidas, 15 ao período diurno e 9 ao noturno, sendo que o período noturno deve começar depois das $22 \mathrm{~h}$ e não deve terminar antes das 7 h do dia seguinte. Como se verifica a seguir, o IPR tornou-se equivalente $\mathrm{L}_{d n}$ ou $D N L$ (Day Night Level).

\section{6 $L_{d n}($ Day Night Level)}

O $L_{d n}$ é uma medida cumulativa da energia total do som, geralmente compilada em uma base anual, e representa uma média logarítmica dos níveis sonoros no local durante um período de 24 horas, com uma penalização de $10 \mathrm{~dB}$ adicionado a todos os sons que ocorram durante o horário noturno (das $22 \mathrm{~h}$ as $7 \mathrm{~h}$ ). A pena de $10 \mathrm{~dB}$ representa a intromissão do ruído adicionado à noite, pois os níveis de som ambiente durante as horas noturnas são, tipicamente cerca de $10 \mathrm{~dB}$ inferiores aos níveis medidos durante o dia, e por causa da irritação associada a distúrbios do sono (FAA, 2011).

De acordo com a NBR 11.415 (ABNT, 1990), o nível de incômodo sonoro medido pelo método $L_{d n}$ é determinado pelo $L_{\text {eq }}$ para $24 \mathrm{~h}$, sendo que no período das $22 \mathrm{~h}$ às $7 \mathrm{~h}$, somam-se $10 \mathrm{~dB}$ a todos os níveis medidos. É definido da seguinte forma: 


$$
L_{d n}=10 \times \log \left[\frac{1}{24}\left(15 \times 10^{\frac{L_{d}}{10}}+9 \times 10^{\frac{\left(L_{n}+10\right)}{10}}\right)\right]
$$

Onde o número 24 corresponde às horas medidas, 15 ao período diurno e 9 ao noturno, sendo que o período noturno deve começar depois das $22 \mathrm{~h}$ e não deve terminar antes das $7 \mathrm{~h}$ do dia seguinte.

\section{$1.7 L_{e q, d e n}$ (indicador do nível de pressão sonora dia-fim-de-tarde-noite)}

A Diretiva Européia 2002/49/EC indica os parâmetros Leq,den (indicador do nível de pressão sonora dia-fim-de-tarde-noite) e $\mathrm{L}_{\text {eq,noite }}$ (indicador do nível de pressão sonora noturno) como indicadores de longo prazo. $\mathrm{O} \mathrm{L}_{\text {eq,den }}$ é definido pela Equação:

$$
L_{e q, \text { den }}=10 \times \log _{10}\left[\frac{1}{24}\left(12 \times 10^{\frac{L_{\text {Aeq, dia }}^{10}}{10}}+4 \times 10^{\frac{L_{\text {Aeq, }, e n t}+5}{10}}+8 \times 10^{\frac{L_{\text {Aeq, noite }}+10}{10}}\right)\right]
$$

O L L eq,dia é o nível de pressão sonora equivalente e contínua referente a um período corresponde às $12 \mathrm{~h}$ avaliado entre $7 \mathrm{~h}$ e $19 \mathrm{~h}$, medido com o filtro de frequências na ponderação $\mathrm{A}$; $\mathrm{L}_{\mathrm{eq}, \text { ent }}$ é o nível de pressão sonora equivalente e contínua referente a um período corresponde às $4 \mathrm{~h}$ avaliado entre $19 \mathrm{~h}$ e $23 \mathrm{~h}$ (entardecer), medido com o filtro de frequências na ponderação $\mathrm{A} ; \mathrm{L}_{\text {eq,noite }}$ é o nível de pressão sonora equivalente e contínua referente a um período corresponde às $10 \mathrm{~h}$ avaliado entre $23 \mathrm{~h}$ e $7 \mathrm{~h}$, medido com o filtro de frequências na ponderação A.

Essa Equação mostra que o indicador $\mathrm{L}_{\mathrm{eq} \text {,den }}$ representa o nível de pressão sonora médio nas $24 \mathrm{~h}$ do dia, com a aplicação de uma ponderação diferenciada para os ruídos emitidos durante o período do anoitecer/entardecer (correção $+5 \mathrm{~dB}$ ) e da noite (correção $+10 \mathrm{~dB}$ ).

\subsection{SEL - Nível de exposição sonora (Sound Exposure Level)}

Vários grupos de pesquisa consideram o SEL como o melhor método para caracterizar o ruído ambiente nas vizinhanças de um aeroporto. Representa a soma de todos os níveis de pressão sonora dentro de um intervalo de interesse, ou seja, SEL é um valor que indica o nível constante, de duração de 1 segundo, que tem igual quantidade de energia que o nível 
equivalente do ruído medido. Este é um índice útil para calcular os níveis sonoros que resultam de qualquer combinação de fontes sonoras (Moraes, E. e N. Lara, 2003).

O SEL não representa o nível sonoro percebido em determinado momento diretamente, mas provê a medida líquida de energia do evento acústico inteiro (EUROPEAN COMMISSION WORKING GROUP, 2010), ou seja, é o nível de um som constante, com uma duração de 1 segundo, o que proporcionaria uma quantidade de energia de som igual à energia do evento em estudo. Pode ser calculado usando a Equação para o nível de pressão sonora equivalente com a duração $(\mathrm{T})$ passando a ser um tempo referenciado $\left(\mathrm{T}_{\text {ref }}\right)$ de 1 segundo. A Equação para a determinação do SEL é, então, expressa da seguinte forma (FICON, 1992 e NBR 11.415):

$$
S E L=10 \times \log \left[\frac{1}{T_{\text {ref }}} \int_{0}^{t} 10^{\frac{L_{A}(t)}{10}} d t\right]=10 \times \log \left[\int_{0}^{t} 10^{\frac{L_{A}(t)}{10}} d t\right]
$$

Onde, $\mathrm{T}_{r e f}$ é igual a 1 segundo e $\mathrm{L}_{A}(t)$ é o nível de ruído na escala $\mathrm{A}$, no instante $t$.

\section{Normas, Portarias e Legislações - Nacionais e Internacionais}

\subsection{Portaria $N^{o} 1.141 / G M 5 / 87$}

A portaria 1.141/GM5 de 1987 estabelece o Plano de Zoneamento de Ruído (PZR) com o intuito de controlar o uso e ocupação do solo nos arredores dos aeroportos, visto que os níveis sonoros nestas áreas são bastante altos (Azevedo e Ortigoza, 2008).

Os ruídos gerados pela operação aeroportuária são estabelecidos pelo Plano de Zoneamento de Ruído (PZR), que é um documento normativo do Comando da Aeronáutica, estabelecendo restrições de uso do solo, constituídas pelo PBZR que definem as áreas de impacto do ruído aeronáutico. Essas áreas podem ser visualizadas na Figura 1 onde: Área 1 - Interior à curva de nível de ruído 1, onde o nível de incômodo sonoro é potencialmente nocivo aos circundantes, podendo ocasionar problemas fisiológicos, devido às exposições prolongadas, sendo o IPR de $75 \mathrm{~dB}(\mathrm{~A})$; Área 2 - Área do Plano de Zoneamento de Ruído, compreendida entre as curvas de nível de ruído 1 e 2 com valores médios do IPR entre $65 \mathrm{~dB}(\mathrm{~A})$ e $75 \mathrm{~dB}(\mathrm{~A})$, onde são registrados níveis de incômodo sonoro moderado; Área 3 - Área do Plano de Zoneamento de 
Ruído, exterior à curva de ruído de nível 2, onde normalmente não são registrados níveis de incômodo sonoro significativos com o valor do IPR sendo inferior a $65 \mathrm{~dB}(\mathrm{~A})$.

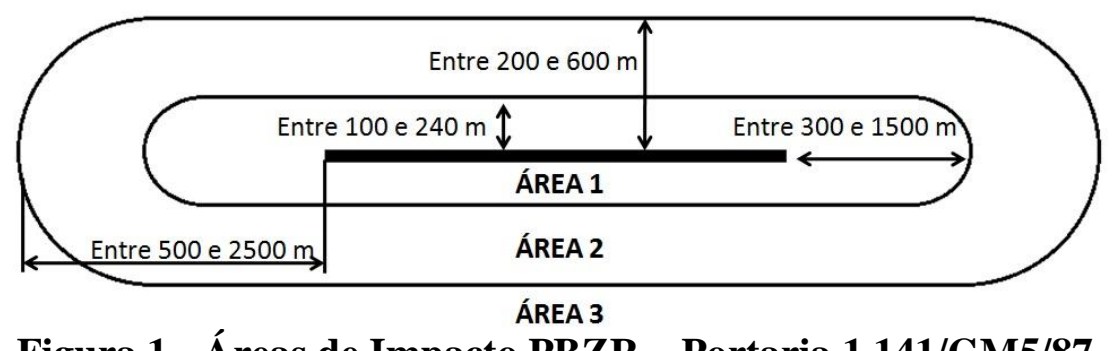

Figura 1 - Áreas de Impacto PBZR - Portaria 1.141/GM5/87

\subsection{NBR 12.859/1993}

Analisa os níveis de incômodo sonoro e fixa condições para gerar curvas isofônicas em áreas sujeitas ao ruído gerado por operações aeronáuticas. O método de avaliação do incômodo sonoro é calculado pelo Índice Ponderado de Ruído (IPR). A Tabela 1 relaciona os valores de IPR conforme a reação da comunidade.

Tabela 1 - Relação entre IPR e a reação das comunidades ${ }^{1}$

\begin{tabular}{l|l}
\hline Valor do IPR & $\begin{array}{l}\text { Reação da Comunidade exposta a este nível de } \\
\text { incômodo }\end{array}$ \\
\hline Menor que 53 IPR & $\begin{array}{l}\text { Nenhuma reclamação é esperada. Ambiente pouco } \\
\text { ruidoso }\end{array}$ \\
\hline Entre 53 IPR E 60 IPR & $\begin{array}{l}\text { É esperado grande volume de reclamações por parte dos } \\
\text { residentes. Ambiente medianamente ruidoso }\end{array}$ \\
\hline Maior que 60 IPR & $\begin{array}{l}\text { São esperadas reclamações generalizadas por parte dos } \\
\text { residentes. É possível ação comunitária em prol da } \\
\text { redução do nível de ruído }\end{array}$ \\
\hline
\end{tabular}

\subsection{NBR 10.856/89}

A NBR 10.856/89 (ABNT) determina o nível efetivo de ruído percebido "Effective Perceived Noise Level" (EPNL), nas medições de ruído de sobrevoo de aeronaves. O EPNL analisa as três propriedades básicas do ruído: nível de pressão sonora, distribuição de frequência e variação no tempo.

\footnotetext{
${ }^{1}$ Fonte: NBR $12.859 / 1993$
} 


\section{$2.4 N B R$ 13.368/95}

A NBR 13.368/95 (ABNT, 1995) prescreve o método para monitoramento de ruídos gerados por aeronaves que verifica a existência do impacto sonoro gerado pelo ruído aeronáutico (Lra) em relação ao ruído de fundo (Lrf), conforme a Tabela 2.

Tabela 2 - Impacto sonoro gerado pelo Lra em relação ao Lrf $^{2}$

\begin{tabular}{l|c}
\hline Impacto sonoro & Lra - Lrf (dB) \\
\hline Desprezível & $<3$ \\
\hline Significativo & $>3$ \\
\hline
\end{tabular}

O valor encontrado para a pressão sonora equivalente (Leq), durante as medições, deve ser comparado com os valores da Tabela 3, visando à avaliação do incômodo gerado pelas operações aeroportuárias.

Tabela 3 - Avaliação do incômodo gerado pelas operações aeroportuárias ${ }^{3}$

\begin{tabular}{l|c|c}
\hline \multicolumn{1}{c|}{ Reclamações esperadas } & Diurno & Noturno \\
\hline Sem reação ou queixa esporádicas & Leq $<65$ & Leq $<55$ \\
\hline $\begin{array}{l}\text { Queixas generalizadas - Possíveis ações da } \\
\text { comunidade }\end{array}$ & $75>$ Leq $>65$ & $\begin{array}{c}65>\text { Leq }> \\
55\end{array}$ \\
\hline Ações comunitárias vigorosas & Leq $>75$ & Leq $>65$ \\
\hline
\end{tabular}

\subsection{NBR 11.415/1990}

A NBR 11.415/1990, estabelece termos e grandezas empregados no ruído aeronáutico e descreve a quantidade máxima de exposição diária permissível dos níveis de ruído que uma pessoa está sujeita, onde o tempo máximo de exposição para um ruído de $85 \mathrm{~dB}(\mathrm{~A})$ é de 8 horas diárias e para um ruído de $115 \mathrm{~dB}(\mathrm{~A})$ é de 7 minutos diários.

\subsection{Regulamento Brasileiro da Aviação Civil (RBAC) $n \stackrel{\circ}{\circ} 161$ - Planos de Zoneamento de}

\section{Ruídos de Aeródromos (PZR)}

Essa resolução define um PZR como um documento que tem como objetivo representar geograficamente a área de impacto do ruído aeronáutico decorrente das operações nos aeródromos e, aliado ao ordenamento adequado das atividades situadas nessas áreas, ser o

\footnotetext{
${ }^{2}$ Fonte: NBR $13.368 / 1995$

${ }^{3}$ Fonte: NBR 13.368/1995
} 
instrumento que possibilita preservar o desenvolvimento dos aeródromos em harmonia com as comunidades localizadas em seu entorno (Resolução n. 202 - ANAC, 2011).

Também estabelece que as curvas de ruído deverão ser calculadas por meio de programa computacional que utilize metodologia matemática apropriada para a geração de curvas, na métrica Ldn, considerando como período noturno, o período compreendido entre $22 \mathrm{~h}$ e $7 \mathrm{~h}$ do horário local. Essa resolução ainda define que:

- O operador de aeródromo deve utilizar o critério apresentado a seguir para definir a obrigatoriedade de aplicação de um PEZR (Plano Específico de Zoneamento de Ruído):

- (1) para aeródromos com média anual de movimento de aeronaves dos últimos 3 (três) anos superior a 7.000 (sete mil), deve ser aplicado um PEZR

○ (2) para os demais aeródromos, é facultado ao operador de aeródromo escolher o tipo de plano a ser elaborado, Plano Básico de Zoneamento de Ruído PBZR ou PEZR.

- A ANAC poderá solicitar a elaboração de um PEZR a qualquer aeródromo. Ressaltase que o PBZR possui curvas de ruído de 75 e 65.

As curvas de ruído são linhas traçadas em um mapa, cada uma representando níveis iguais de exposição ao ruído. As cinco curvas de ruído que compõem o PEZR, na métrica $\mathrm{L}_{d n}$, são (RBAC 161, 2011):

- Curva de Ruído de 85 é a linha traçada a partir da interpolação dos pontos que apresentam nível de ruído médio dia-noite de $85 \mathrm{~dB}$.

- Curva de Ruído de 80 é a linha traçada a partir da interpolação dos pontos que apresentam nível de ruído médio dia-noite de $80 \mathrm{~dB}$.

- Curva de Ruído de 75 é a linha traçada a partir da interpolação dos pontos que apresentam nível de ruído médio dia-noite de $75 \mathrm{~dB}$.

- Curva de Ruído de 70 é a linha traçada a partir da interpolação dos pontos que apresentam nível de ruído médio dia-noite de $70 \mathrm{~dB}$.

- Curva de Ruído de 65 é a linha traçada a partir da interpolação dos pontos que apresentam nível de ruído médio dia-noite de $65 \mathrm{~dB}$. 
Para exemplificar, a Figura 2 mostra a simulação elaborada com a metodologia proposta pela RBAC 161, para o Aeroporto Internacional de Brasília (AIB). O mapa foi gerado com auxílio do software INM 7.0c (Integrated Noise Model) para operação mensal das principais empresas aéreas da aviação civil, tendo como base o mês de maio de 2012.

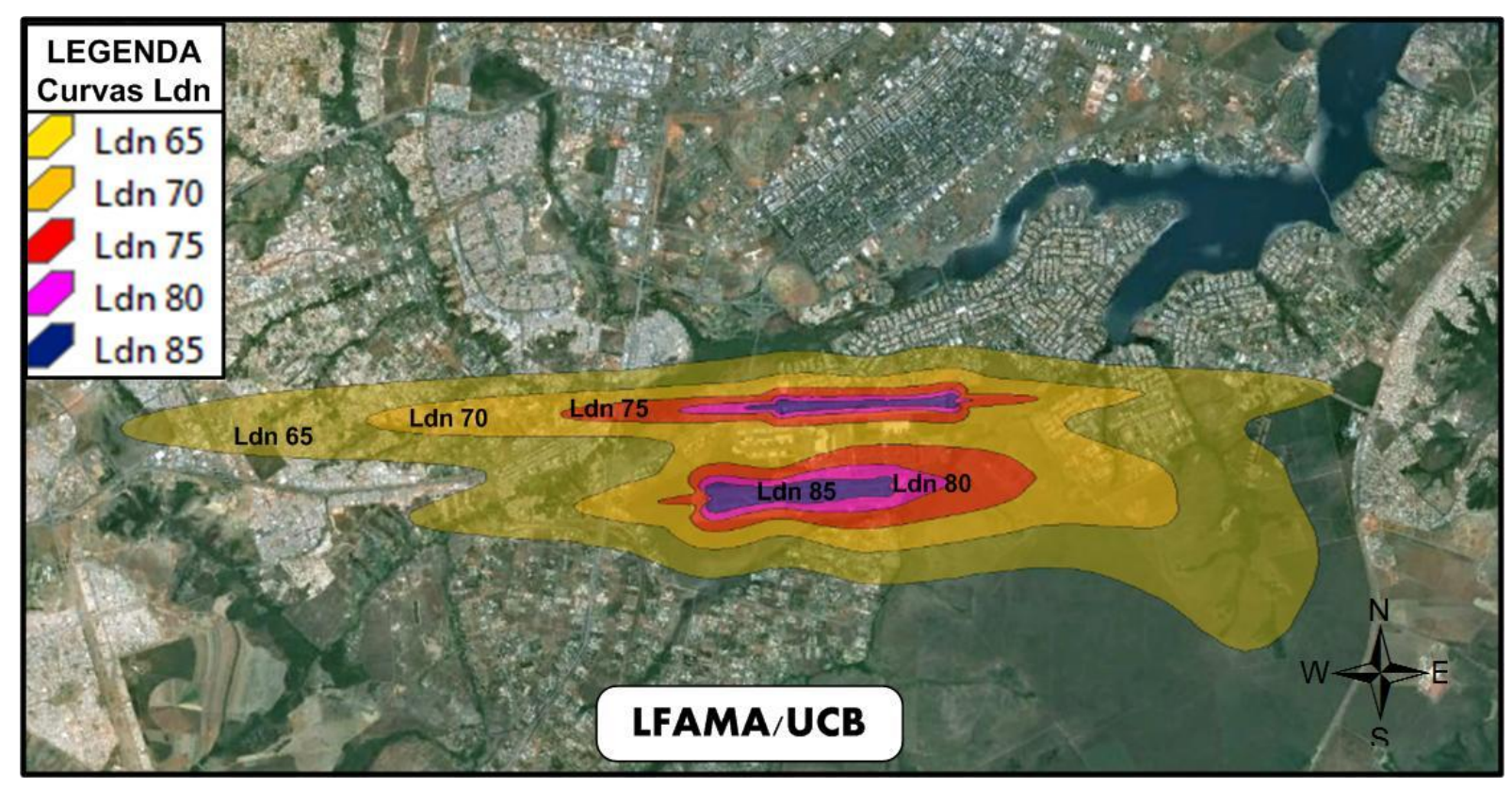

Figura 2 - Mapa de ruídos do AIB para a métrica $\mathrm{L}_{d n \cdot}{ }^{4}$

\subsection{Legislação Ambiental - Resolução CONAMA n. 001/1990 e NBR 10.151}

A resolução dispõe sobre a emissão de ruídos, em decorrência de qualquer atividade industrial, comercial, social ou recreativa, inclusive as de propaganda política. Para a resolução ter efeito de aplicabilidade, as medições deverão ser efetuadas conforme a NBR 10.151 (ABNT) que fixa os níveis de ruído superiores que são prejudiciais à saúde e estabelece condições exigíveis para a avaliação da aceitabilidade do ruído em áreas habitadas, visando o conforto das comunidades e especifica um método para a medição de ruído.

O método de avaliação envolve as medições do nível de pressão sonora equivalente $\left(\mathrm{L}_{\mathrm{eq}}\right)$, ponderados em $\mathrm{dB}(\mathrm{A})$. Estabelece níveis de ruídos em seis áreas distintas, sendo cinco áreas urbanas e uma área rural (Tabela 4).

\footnotetext{
${ }^{4}$ Fonte: LFAMA/UCB (Laboratório de Física Aplicada ao Meio Ambiente - Universidade Católica de Brasília).
} 
Tabela 4 - Nível de critério de avaliação para ambientes externos, em $\mathrm{dB}(\mathrm{A})^{5}$

\begin{tabular}{lcc}
\hline \multicolumn{1}{c}{ Tipos de áreas } & Diurno & Noturno \\
\hline Áreas de sítios e fazendas & 40 & 35 \\
Área estritamente residencial urbana ou de hospitais ou de escolas & 50 & 45 \\
Área mista, predominantemente residencial & 55 & 50 \\
Área mista, com vocação comercial e administrativa & 60 & 55 \\
Área mista, com vocação recreacional & 65 & 55 \\
Área predominantemente industrial & 70 & 60 \\
\hline
\end{tabular}

Atualmente, a NBR 10.151/2000 encontra-se em processo de reformulação.

\subsection{Experiências Internacionais}

O Departamento de Transportes do Canadá (Transport Canada) utiliza o NEF (Noise Exposure Forecast) para fornecer uma medida do ruído das aeronaves nas proximidades dos aeroportos. O principal objetivo da utilização do NEF é colaborar com os planejadores de aviação e os responsáveis pelo desenvolvimento das áreas adjacentes aos aeroportos, para implementar práticas de zoneamento inteligente e gerenciar adequadamente o uso do solo nas imediações dos aeroportos. Além disso, essa métrica permite prever a resposta de uma comunidade ao ruído das aeronaves. Se o nível de NEF é maior que 35, as queixas tendem a ser elevados. Qualquer valor acima de 25 é susceptível de produzir algum nível de aborrecimento (TC, 2011a).

O grande número de cálculos matemáticos necessários para a construção de curvas isofônicas com o NEF, requer o uso de técnicas computacionais para a elaboração de mapas. Assim, a determinação das curvas de ruído, com o uso do NEF, é estritamente um procedimento de cálculo numérico. Desta forma, técnicas de modelagem computacionais fornecem o único meio prático de construção dessas curvas (TC, 2011b).

\footnotetext{
${ }^{5}$ Fonte: NBR 10.151/2000
} 
O Departamento de Habitação e Desenvolvimento Urbano dos Estados Unidos (US HUD) utiliza o NEF e o $L_{d n}$ para monitoramento do ruído. O documento intitulado Noise Guidebook foi preparado para servir de referência básica para o pessoal de campo do US HUD, que são responsáveis pela implementação das políticas relacionadas à ruídos do Departamento (US HUD, 2011). Para análise de ruído aeroviário o Federal Aviation Regulation (FAR) determina o Day Night Level - Nível de ruído médio $\left(L_{d n}\right)$, como a métrica primária do FAA (Federal Aviation Administration) para medir a exposição cumulativa do indivíduo à energia sonora resultante de atividades aéreas. Dessa forma, os principais orgãos do governo americano relacionados ao monitoramento de ruído trabalham com o NEF e o $L_{d n}$.

$\mathrm{Na}$ Índia também utiliza-se $L_{d n}$ e o NEF para se estabelecer o zoneamento sonoro e efetuar o monitoramento de regiões próximas a aeroportos. O governo indiano prevê que o ruído das aeronaves pode afetar seriamente as pessoas e por esta razão, recomenda que as residências sejam construídos a partir da curva NEF 35 (EIA Guidance Manual, 2010 e CPCB, 2008).

Já na África do Sul as métricas utilizadas são $\mathrm{L}_{R d n}$ que representa o impacto do ruído de aeronaves atualmente prescritos pelas normas nacionais sul-africanos. Essa métrica é idêntica ao $L_{d n}$, ou seja, com uma ponderação de $10 \mathrm{~dB}$ para voos noturnos. O que muda é o período noturno que é definido como sendo de $22 \mathrm{~h}$ as $06 \mathrm{~h}$. Também se utiliza nesse país a métrica Noisiness Index (NI) para a avaliação do nível de incômodo sonoro. Essa métrica representa o ruído aeroviário como uma ponderação de $5 \mathrm{~dB}$ adicionado aos voos que operam entre $22 \mathrm{~h}$ e 24h, e $10 \mathrm{~dB}$ para voos entre as $24 \mathrm{~h}$ e as 06h (Goldschagg, P., 2007).

Na Comunidade Européia, a Diretiva 2002/49/CE, recomenda, no Anexo II, aos Estados Membros, que não dispõem de métodos de cálculo nacionais ou que pretendem mudar o método de cálculo, a utilização das métricas $\mathrm{L}_{\mathrm{eq}, \mathrm{den}} \mathrm{e} \mathrm{L}_{\mathrm{n}}$ para o ruído gerado pelo setor industrial, pelas aeronaves e pelo tráfego rodoviário e ferroviário. 


\section{Resultados e Discussões}

Nesta seção, efetua-se a comparação das metodologias e métricas acústicas empregadas em cada uma das normas e legislações avaliadas. Essa análise comparativa possui por objetivo identificar abordagens comuns, ou diferentes, adotadas pelos países estudados com relação à avaliação do ruído aeronáutico. Inicialmente, busca-se identificar as divergências entre as normas brasileiras e a Tabela 5 resume as principais características analisadas.

Percebe-se que a ABNT recomenda quatro normas para ruído aeronáutico: NBR 10.856/1989, NBR 11.415/ 1990, NBR 12.859/1993 e NBR 13.368/1995. A NBR 11.415 somente define os termos e grandezas empregados na área de ruído aeronáutico. A NBR 12.859/1993 define procedimentos para a avaliação do impacto sonoro gerado pelo ruído aeroportuário e utiliza como métrica principal o IPR. Já a NBR 10.856 fixa as condições exigíveis para a determinação do nível efetivo de ruído percebido - EPNL (Effective Perceived Noise Level), nas medições de ruído de aeronaves.

A NBR 13.368/1995 prescreve o método para monitoração de ruídos gerados por aeronaves que verifica a existência do impacto sonoro gerado pelo ruído aeronáutico (Lra) em relação ao ruído de fundo (Lrf). Em consonância com a portaria 1.141/GM5 (1987) tem-se a NBR 12.859 de 1993, que analisa os níveis de incômodo sonoro e fixa condições para gerar curvas isofônicas em áreas sujeitas ao ruído gerado por operações aeronáuticas.

Ressalta-se que a portaria 1.141/GM5 (1987) e as normas NBR's $11.415,10.856$ e 12.859 se complementam e indicam a métrica e a metodologia para se verificar o nível de incômodo sonoro em áreas adjacentes a aeroportos bem como o método de zoneamento urbano e sonoro a ser seguido para a implantação de um aeródromo. No entanto, a NBR 13.368, de 1995, propõem para monitoração medir os valores do nível de ruído equivalente contínuo $\left(\mathrm{L}_{\mathrm{eq}}\right)$, por um período mínimo de $1 \mathrm{~h}$, sem interrupção, durante o período considerado mais crítico. Também expressa uma tabela onde estabelece a avaliação do incômodo gerado pelas operações aeroportuárias tomando como referência o $\mathrm{L}_{\mathrm{eq}}$. Aqui se verifica uma incoerência metodológica entre a portaria 1.141/GM5 (1987) e NBR 12.859 com a NBR 10.856. 
Tabela 5 - Tabela resumo das principais normas e legislações abordadas no artigo

\begin{tabular}{|c|c|c|c|c|}
\hline $\begin{array}{c}\text { Normas e } \\
\text { Legislações }\end{array}$ & Mês / Ano & Aplicação & $\begin{array}{l}\text { Métrica } \\
\text { Acústica }\end{array}$ & Observações \\
\hline $\begin{array}{c}\text { Portaria } \\
\text { 1.141/GM5 }\end{array}$ & $\begin{array}{c}\text { Dezembro } \\
\text { de } 1987\end{array}$ & $\begin{array}{l}\text { Dispõe sobre Zonas de Proteção e } \\
\text { Aprova o Plano Básico de Zona de } \\
\text { Proteção de Aeródromos, o Plano } \\
\text { Básico de Zoneamento de Ruído, o } \\
\text { Plano Básico de Zona de Proteção de } \\
\text { Helipontos e o Plano de Zona de } \\
\text { Proteção de Auxílios à Navegação } \\
\text { Aérea e dá outras providências. }\end{array}$ & IPR & $\begin{array}{l}\text { Define o IPR como sendo } \\
\text { uma unidade de avaliação do } \\
\text { incômodo sonoro. Não faz } \\
\text { referência a nenhuma norma } \\
\text { específica, mas a definição } \\
\text { do IPR é a mesma indica na } \\
\text { NBR } 11.415 / 1990 \text {. }\end{array}$ \\
\hline $\begin{array}{c}\text { NBR } \\
10.856\end{array}$ & $\begin{array}{c}\text { Agosto de } \\
1989\end{array}$ & $\begin{array}{l}\text { Fixa as condições exigíveis para a } \\
\text { determinação } \\
\text { do nível efetivo de ruído percebido } \\
\text { "Effective Perceived Noise Level" } \\
\text { (EPNL), nas medições de ruído de } \\
\text { sobrevoo de aeronaves. }\end{array}$ & EPNL & \\
\hline $\begin{array}{c}\text { NBR } \\
11.415\end{array}$ & $\begin{array}{c}\text { Novembro } \\
\text { de } 1990\end{array}$ & $\begin{array}{l}\text { Esta norma define os termos e } \\
\text { grandezas na área de ruído } \\
\text { aeronáutico. }\end{array}$ & $\begin{array}{l}\mathrm{IPR}, \mathrm{L}_{d n}, \\
\mathrm{~L}_{\mathrm{eq}}, \\
\mathrm{NEF}, \\
\text { EPNL, } \\
\text { etc. }\end{array}$ & $\begin{array}{l}\text { Define vários conceitos } \\
\text { utilizados na pesquisa em } \\
\text { acústica. }\end{array}$ \\
\hline $\begin{array}{c}\text { NBR } \\
12.859\end{array}$ & $\begin{array}{c}\text { Maio de } \\
1993\end{array}$ & $\begin{array}{l}\text { Fixa as condições exigíveis para gerar } \\
\text { curvas isofônicas e analisar os níveis } \\
\text { de incômodo sonoro em função das } \\
\text { áreas sujeitas ao ruído gerado por } \\
\text { operações aeronáuticas. }\end{array}$ & IPR & $\begin{array}{l}\text { Para a aplicação dessa norma } \\
\text { é necessário consultar a NBR } \\
10.856 \text { e NBR } 11.415 \text {. }\end{array}$ \\
\hline $\begin{array}{c}\text { NBR } \\
13.368\end{array}$ & $\begin{array}{c}\text { Maio de } \\
1995\end{array}$ & $\begin{array}{lllll}\text { Prescreve } & \text { o } & \text { método } & \text { para } & \text { a } \\
\text { monitoração de } & \text { ruído gerado por } \\
\text { aeronaves. } & & & & \end{array}$ & $\mathrm{L}_{\mathrm{eq}}$ & $\begin{array}{l}\text { Para a aplicação dessa norma } \\
\text { é necessário consultar: NBR } \\
10.268 \quad \text { (Ruído nas } \\
\text { dependências de terminais de } \\
\text { passageiros aeroportuários) e } \\
\text { NBR 11.415. }\end{array}$ \\
\hline $\begin{array}{l}\text { Resolução } \\
\text { Conama } \\
001\end{array}$ & $\begin{array}{c}\text { Março de } \\
1990\end{array}$ & $\begin{array}{l}\text { Dispõe sobre a emissão de ruídos, em } \\
\text { decorrência de qualquer atividade } \\
\text { industrial, comercial, social ou } \\
\text { recreativa, inclusive as de propaganda } \\
\text { política. }\end{array}$ & & Reporta-se à NBR 10.151 \\
\hline
\end{tabular}


A métrica IPR é indicada para um período de medição de $24 \mathrm{~h}$ e o $\mathrm{L}_{\mathrm{eq}}$, na NBR 13.368 , somente para 1 hora de medição. A Resolução CONAMA nº 1 de 1990 é uma norma ambiental e reporta-se à NBR 10.151, que utiliza como principal métrica o $\mathrm{L}_{\mathrm{eq}}$ e fixa as condições exigíveis para avaliação da aceitabilidade do ruído em comunidades. Os níveis de critério estabelecidos nessa norma são obtidos por meio do cálculo do $\mathrm{L}_{\mathrm{eq}}$ assim como o estabelecido na NBR 13.368. Entretanto, a NBR 10.151 não estabelece um tempo de medição de uma hora e apresenta limites de níveis de pressão sonora para áreas residenciais, em ambientes urbanos, mais restritivos aos propostos pela NBR 13.368.

No trabalho de Carvalho Jr. E. e Garavelli. S (2010) compararam-se os limites estabelecidos na NBR 10.151 com os impostos na portaria 1.141/GM5 (1987) e na norma NBR 12.859 . Dessa comparação, tem-se que para atender a legislação ambiental, o IPR, da isofônica 2, deveria variar de 43, em áreas de sítios e fazendas, a 70 em áreas predominantemente industriais. Três áreas de uso misto (residenciais e comerciais) e uma área de uso estritamente residencial foram analisadas. Calculou-se o IPR com os valores do $\mathrm{L}_{\mathrm{eq}}$ estabelecidos na NBR 10.151 e como resultado verificou-se que em todas as áreas, os valores do IPR apontavam para uma possível reação por parte dos moradores, pois segundo a NBR 12.859 para um IPR superior a $60 \mathrm{~dB}(\mathrm{~A})$ são esperadas reclamações generalizadas por parte dos residentes sendo possível ação comunitária em prol da redução do nível de ruído.

O Departamento de Transporte do Canadá estabelece o uso do NEF em suas normas de avaliação do ambiente sonoro. Ocorre a mesma recomendação no Departamento de Habitação e Desenvolvimento Urbano dos Estados Unidos que também indica o uso do $L_{d n}$. O FAA também estabelece o $L_{d n}$ para avaliação do ruído aeronáutico em zonas próximas a aeródromos. Ressalta-se, que nesses países não utiliza-se somente o $L_{d n}$, mas também o NEF como métrica para a elaboração de mapas acústicos.

A Transport Canada recomenda que edificações residenciais somente são permitidas em áreas próximas a aeroportos se os níveis de ruído forem iguais a um NEF de 30 ou menos. Isto é aproximadamente equivalente a um $L_{d n} 61$ e é um pouco menor que o $L_{d n} 65$ limite nos EUA. Ressalta-se que regiões que apresentem $L_{d n}$ acima de 75 são inadequadas para o uso residencial. Isso ocorre também no Brasil, onde o limite estabelecido para o $L_{d n}$ é de $65 \mathrm{~dB}(\mathrm{~A})$ para a área III. 
Entretanto, cabe chamar a atenção para a utilização da métrica $\mathrm{L}_{d n}$ proposta pelo FAA, Transport Canada e pelo Brasil a partir do ano passado. O estudo realizado por Heleno (2010) constatou que o $\mathrm{L}_{d n}$ não seria uma boa métrica para o zoneamento, já que esta métrica deve ser baseada nas atividades diurnas e noturnas. O efeito causado pelo ruído durante o dia ou durante a noite influi de maneira diferente na realização de atividades cotidianas. Assim, o uso do $\mathrm{L}_{d n}$ para o zoneamento aeroportuário pode conduzir a resultados diferentes dos níveis sonoros diurnos e noturnos dependendo do número de operações do aeroporto no período diurno e noturno (Heleno, 2010).

Os estudos de Carvalho Jr. e Garavelli, S. (2010) e Rocha e Slama (2008) também apontam para existência de conflitos entre a métrica proposta na NBR 10.151 e a métrica $\mathrm{L}_{d n}$. A métrica $\mathrm{L}_{d n}$ é uma métrica calculada para um período de 24 horas enquanto que as métricas na NBR 10.151 são calculadas sobre os períodos diurno e noturno. No trabalho de Carvalho Jr, E., Garavelli, S. e Maroja, A. (2012) verificou-se que a percepção de incômodo sonoro, por ruído aeroviário, melhor se correlaciona com os limites indicados na NBR 10.151.

Sendo assim, não existe uma relação biunívoca entre as duas famílias de métricas. Para um mesmo valor de $\mathrm{L}_{d n}$, encontra-se diversos valores de $\mathrm{L}_{\mathrm{d}}$ e $\mathrm{L}_{\mathrm{n}}$ dependendo do aeroporto. Além disso, existem basicamente três grandes conflitos entre o PZR, regulamentado pela Portaria 1.141, e os critérios de zoneamento definidos pela NBR 10.151: as métricas divergentes, o número de áreas estabelecidas em cada um dos zoneamentos e os níveis de ruído considerados (Rocha e Slama, 2008).

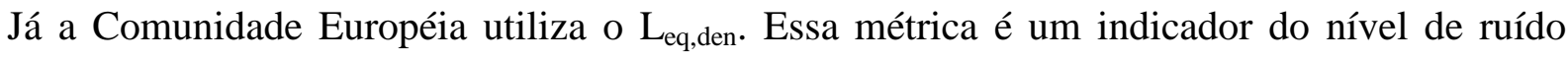
global ao longo do período dia/entardecer/noite, utilizado para qualificar o desconforto associado à exposição ao ruído. O Ln é um indicador do nível sonoro durante a noite, que qualifica as perturbações do sono. Os indicadores de ruído Lden e Ln são utilizados para estabelecer os mapas de ruído estratégicos.

O SEL é utilizado para medir o ruído associado a eventos individuais e é muito utilizado para dimensionar a interferência no sono ocasionada pelo ruído aeronáutico. A Federal Interagency Committe on Aviation Noise (FICAN, 1997) usa o SEL para prever o percentual de pessoas que iriam sofrer distúrbios no sono decorrentes da passagem de uma aeronave. $\mathrm{O}$ 
SEL também é usado na Comunidade Européia, no período noturno, para avaliação dos possíveis picos de ruídos advindos da passagem de aeronaves.

\section{Conclusão}

Nesse trabalho, apresenta-se uma análise comparativa entre as principais normas e legislações que avaliam o ruído proveniente da operação de aeronaves. Comparou-se as metodologias adotadas e as métricas acústicas utilizadas tendo como referência o contexto brasileiro. Experiências internacionais também foram consideradas com o intuito de determinar semelhanças, diferenças ou relação entre as estratégias adotadas por diferentes países para o monitoramento e controle do ruído aeronáutico.

Da análise realizada foi possível verificar que existem divergências entre as normas e portarias, principalmente com relação à métrica acústica empregada para monitoração e caracterização do nível de incômodo sonoro gerado por tráfego aéreo. A portaria 1.141/GM5 (1987) e as normas NBR's $11.415,10.856$ e 12.859 se complementam e indicam a métrica IPR para a elaboração de curvas isofônicas.

No entanto, o IPR foi atualizado em 1994 tornando-se uma métrica equivalente ao $\mathrm{L}_{d n}$. Somente em 2011 a Resolução ANAC nº. 202 estabelece para os operadores de aeródromos, os requisitos de elaboração e aplicação do PZR definindo critérios para o adequado ordenamento das atividades situadas em áreas próximas a aeroportos. Essa resolução pretende tornar o PZR um instrumento que possibilite o desenvolvimento dos aeródromos em harmonia com as comunidades localizadas em seu entorno. Além disso, estabelece que as curvas de ruído devam ser calculadas por meio de programa computacional com uso da métrica $\mathrm{L}_{d n}$. Dessa forma, sugere-se que trabalhos futuros reportem sempre a essa nova resolução.

Comparando as normas brasileiras com as utilizadas em outros países, verifica-se uma adEquação quanto à métrica $L_{d n}$. No entanto, os países estudados utilizam métricas complementares como o NEF e o SEL na América do Norte e o Leq,den na Europa. No sentido de melhor entender os impactos ao ambiente sonoro de regiões próximas a aeródromos, seria importante a realização de estudos com as métricas NEF, SEL e $\mathrm{L}_{\text {eq,den }}$ no contexto brasileiro. Os resultados desses estudos podem apontar para a necessidade de se utilizar alguma dessas 
métricas no auxílio ao planejamento do uso e ocupação do solo em áreas circunvizinhas a aeroportos bem como no monitoramento do ruído aeronáutico.

Por fim, esse estudo busca contribuir para um melhor entendimento das principais métricas acústicas atualmente empregadas para a avaliação do ruído aeroviário, em áreas circunvizinhas a aeroportos. Ao permitir identificar contradições e relações entre as diversas legislações, também possui potencial de contribuir para o aprimoramento dos planos de zoneamento de ruído em aeródromos proporcionando, assim, a minimização dos impactos inerentes à realização das atividades aeroportuárias assegurando condições mais adequadas de relacionamento entre os operadores de aeródromos e a comunidade.

\section{Referências}

Associação Brasileira de Normas Técnicas - ABNT (2000) NBR 10.151: Avaliação do Ruído em Áreas Habitadas, Visando o Conforto da Comunidade. Rio de Janeiro.

Associação Brasileira de Normas Técnicas - ABNT (1989) NBR 10.856: Determinação do Nível Efetivo de Ruído Percebido (EPNL) de Sobrevoo de Aeronaves. Rio de Janeiro.

Associação Brasileira de Normas Técnicas - ABNT (1990) NBR 11.415: Ruído Aeronáutico. Rio de Janeiro.

Associação Brasileira de Normas Técnicas - ABNT (1993) NBR 12.859: Avaliação do Impacto Sonoro Gerado por Operações Aeronáuticas. Rio de Janeiro.

Associação Brasileira de Normas Técnicas - ABNT (1995) NBR 13.368: Ruído Gerado por Aeronaves - Monitoração. Rio de Janeiro.

Azevedo, J. C. e Ortigoza, S. G. (2008) Instalação e ampliação de aeroportos e seus impactos sócioespaciais em cidades paulistas: o caso de Ribeirão Preto. $7^{\circ}$ Simpósio de Transporte Aéreo SITRAER, Rio de Janeiro.

Babisch, W., Houthuijs, D., Pershagen, G., Cadum, E., Katsouyanni, K., Velonakis, M. (2009) Annoyance due to aircraft noise has increased over the years-results of the HYENA study. Environmental International, vol. 35, pp. 1169-1176.

Bistafa, S. (2006) Acústica Aplicada ao Controle do Ruído. São Paulo: Editora Edgard Blücher.

Bradley, J. S. (1996) NEF Validation Study: (3) Final report, NRC Contract Report to Transport Canada.

Bressane, A., Mochizuki, P. S., Gobbi, N. e Carvalho, M. D. (2008) Legislação Aplicável à Poluição Sonora Urbana: Um Estudo das Normas e Diretrizes Disciplinares. Holos Environmental, vol. 8, n. 2, pp. 132.

Campos Neto, C. A. S. e Souza, F. H. (2010) Aeroportos no Brasil: investimentos recentes, perspectivas e preocupações. Nota Técnica IPEA, n. 5. Rio de Janeiro: Instituto de Pesquisa Econômica Aplicada.

Carvalho Jr, E. B. e Garavelli, S. (2010) Avaliação do ruído aeronáutico em regiões circunvizinhas ao Aeroporto Internacional de Brasília. XXIII Encontro da Sociedade Brasileira de Acústica, Salvador. 
Carvalho Jr, E. B., Garavelli, S. L. e Maroja, A. M. (2012) Análise dos efeitos do ruído aeronáutico em zonas residenciais circunvizinhas ao Aeroporto Internacional de Brasília. Journal of Transport Literature, vol. 6, n. 4, pp. 59-81. 2012.

Central Pollution Control Board - CPCB (2008) Requirement and Procedure for Monitoring Ambient Noise Level due to Aircrafts. Ministry of Environmental \& Forests, Government of India. Documento técnico CPCB. Disponível em www.cpcb.nic.in.

Comunidade Europeia, (2002) Relativa à avaliação e gestão do ruído ambiente. Diretiva 2002/49/EC. Disponível em eur-lex.europa.eu.

Decreto n. 89.431 (1984) Plano básico de zoneamento de ruído e planos específicos de zoneamento de ruído. Diário Oficial da União, Brasília, DF, pp. 3673.

EIA Guidance Manual (2010) Environmental Impact Assessment Guidance Manual for building, construction, townships and area development projects. Ministry of Environmental \& Forests, Government of India.

Federal Aviation Administration - FAA (sd) Noise and its Effect on People. Documento técnico FAA. Disponível em: www.faa.gov.

Federal Interagency Committe on Aviation Noise - FICAN (1997) Effects of Aviation Noise on Awakenings from Sleep. Documento técnico FICAN. Disponivel em: www.fican.org.

Federal Interagency Committee on Noise - FICON (1992) Federal Agency Review of Selected Airport Noise Analysis Issues, Report for the Department of Defense, Washington, DC.

Gerges, S. N. Y. (2000) Ruído: fundamentos e controle, Florianópolis: NR Editora.

Goldschagg, P. (2007), Airport Noise in South Africa - prediction models and their effects on land-use planning. Dissertation presented in fulfillment of the requirements for the degree of Doctor of Philosophy at the University of Stellenbosch, South Africa.

Group, W. A. (2003) Nighttime noise criteria and land-use guidelines for the city of high point. Wyle Report. Arlington, Virginia.

Heleno, T. A. (2010) Uma Nova Metodologia de Zoneamento Aeroportuário com o Objetivo de Reduzir o Encroachment e os Efeitos Adversos do Ruído. Dissertação (Mestrado em Engenharia Mecânica), Universidade Federal do Rio de Janeiro, Rio de Janeiro.

Instituto de Aviação Civil - IAC (1981). Métodos de avaliação dos níveis de ruído e de incômodo gerados pela operação de aeronaves em aeroportos. Ministério da Aeronáutica. Rio de Janeiro, Brasil.

Jarup, L., Dudley, M. L., Babisch, W., Houthuijs, D., Swart, W., Pershagen, G., Bluhm, G., Katsouyanni, K., Velonakis, M., Cadum, E. e Vigna-Taglianti, F. (2005) Hypertension and Exposure to Noise near Airports (HYENA): Study Design and Noise Exposure Assessment. Environmental Health Perspectives, vol. 113, pp. 1473-1478.

Moraes, E. M. L. e Lara, N. A. (2003) Método de Elaboração de Mapa de Ruído com Utilização de Variáveis Física e Psicológicas: o Exemplo de Belém-Brasil. $34^{\circ}$ Congresso Tecniacústica, Bilbao.

Nunes, M. e Ribeiro, H. (2008) Interferências do ruído do tráfego urbano na qualidade de vida: Zona residencial de Brasília-DF. Caderno Metrópoles, vol. 19, pp. 319-338.

Nunes, M. F. O. e Sattler, M. A. (2004) Percepção do ruído aeronáutico em escolas da zona I do PEZR do Aeroporto Internacional Salgado Filho. Engevista, vol. 6, n. 3, pp. 5-24.

Pepper, C. B., Nascarella, M. A. e Kendal, R. J (2003) A review of the effects or aircraft noise on widlife na humans, current control mechanisms, and the need for further study. Environmental Management, vol. 32, pp. 418-432. 
Ministério da Aeronáutica - MAer (1987) Dispõem sobre zonas de proteção e aprova o Plano Básico de Zona de Proteção de Aeródromos, o Plano Básico de Zoneamento de Ruído, o Plano Básico de Proteção de Helipontos e o Plano de Zona de Proteção de Auxílios à Navegação Aérea e dá outras providências. Portaria MAer. n. 1.141/GM5, Brasil.

Regulamento Brasileiro da Aviação Civil - RABC 161 (2011) Planos de Zoneamento de Ruído de Aeródromos. Aprovado na Resolução n. 202, de 28 de setembro de 2011, publicado no Diário Oficial da União de 29 de setembro de 2011, Seção 1.

Resolução Conselho Nacional do Meio Ambiente - CONAMA (1990) Dispõe sobre a Emissão de Ruídos, em decorrência de quaisquer atividades industriais, comerciais, sociais ou recreativas, determinando padrões, critérios e diretrizes. Resolução CONAMA n. 1/90.

Rocha, R. e Slama, J. (2008) AdEquação do zoneamento urbano ao zoneamento sonoro dos aeroportos. $7^{\circ}$ Simpósio de Transporte Aéreo - SITRAER, Rio de Janeiro.

Transport Canada - TC (2011a) Aircraft Noise Management. Documento técnico não publicado. Disponivel em www.tc.gc.ca.

Transport Canada - TC (2011b) Part IV - Aircraft Noise. 4.1 General. Documento técnico não publicado. Disponivel em www.tc.gc.ca.

U.S. Department of Housing and Urban Development - US HUD (sd) The Noise Guidebook. Documento técnico US HUD. Disponível em: portal.hud.gov.

Valim, F. C. (2006) Análise das Diferentes Formas de Atenuação do Ruído Aeronáutico. Dissertação (Mestrado em Transportes), Universidade de Brasília, Brasília.

World Health Report - WHO (2001) Occupational and community noise. Documento técnico não publicado. Disponível em www.who.int. 